\title{
Effects of Differences in Problem-Based Learning Course Length on Academic Motivation and Self-Directed Learning Readiness in Medical School Students
}

\author{
So Jung Yune', Sun Ju $\mathrm{Im}^{2}$, Sun Hee Lee ${ }^{2}$, Sun Yong Baek ${ }^{2}$ and Sang Yeoup Lee \\ ${ }^{1}$ Center for Teaching \& Learning, Pusan National University, ${ }^{2}$ Medical Education Unit and Medical \\ Research Institute, Pusan National University Medical School, and ${ }^{3}$ Family Medicine Clinic, Pusan \\ National University Yangsan Hospital, Yangsan, Korea
}

\section{문제바탕학습의 실시기간이 의학전문대학원 학생들의 학습동기와 자기주도학습 준비도에 미치는 영향}

${ }^{1}$ 부산대학교 교수학습지원센터, ${ }^{2}$ 부산대학교 의학전문대학원 의학교육실 및 의학연구소,

${ }^{3}$ 양산부산대학교병원 가정의학클리닉

윤소정, 임선주 ${ }^{2}$, 이선희 $\left.\right|^{2}$, 백선용 $^{2}$, 이상엽 ${ }^{2,3}$

Purpose: Problem-based learning (PBL) is an educational approach in which complex authentic problems serve as the context and stimulus for learning. PBL is designed to encourage active participation during learning. The goal of this study was to study the effects of PBL on academic motivation and self-directed learning readiness in medical school students.

Methods: The subjects of this study were 190 students in the 1st and 2nd grade of medical school. The period of the PBL course was two weeks for Year 1 and five weeks for Year 2 students. Students completed one module over one week. Academic motivation tests and self-directed learning readiness tests were performed before and after the PBL course. The differences between the two groups were analyzed using paired t-test and repeated measures MANCOVA.

Results: PBL had positive effects on academic self-efficacy (self-control efficacy, task-level preference) and academic failure tolerance (behavior, task-difficulty preference) as academic motivation. PBL had a mildly positive effect on self-directed learning readiness. In addition, the five-week PBL course had greater positive effects on academic motivation than the two-week course but not with regard to self-directed learning readiness.

Conclusion: Medical students engage in academic motivation and self-directed learning readiness during PBL, suggesting that the five-week PBL course has greater positive effects than the two-week course. Future studies are needed to confirm the most effective periods of $\mathrm{PBL}$.

Key Words: Problem-based learning, Learning, Motivation, Program evaluation

Received: December 8, 2009 - Accepted: February 8, 2010 Corresponding Author: Sang Yeoup Lee

Medical Education Unit, Pusan National University School of Medicine, Beomeo-ri Mulgeum-eup, Yangsan, 626-870, Korea

TEL) 010-9134-5959 FAX) 051-510-8125 E-mail) saylee@pnu.edu
Korean J Med Educ 2010 Mar; 22(1): 23-31. doi: $10.3946 /$ kjme.2010.22.1.23.

(C) The Korean Society of Medical Education. All rights reserved. 


\section{서론}

지식기반사회는 문제의 속성을 이해하고, 문제 해결의 다 양한 방안을 활용하며, 쏟아지는 정보를 자원으로 환원시킬 수 있는 인간의 능력을 요구하고 있다. 이에 따라 의학교육에 서는 급증하고 있는 방대한 의학 지식을 적절히 활용하여 문 제를 능동적으로 해결할 수 있는 의사를 양성하기 위하여 기 존의 주입식, 강의식, 암기식 교육에서 탈피하고 과제 혹은 수 기위주의 교육과 문제해결위주의 교수법 등 다양한 방법이 사용되고 있다. 그 중 문제바탕학습(problem-based learning, PBL)은 학습자의 적극적인 참여와 자기주도학습을 강조 하는 학습이라는 점에서 국내외 여러 의학교육현장에서 많이 활용되고 있다. PBL은 학습자의 인지적 능력에 의한 지식 습 득뿐 아니라, 학업에 대한 자기주도성[1,2]과 학습동기[3,4,5] 등의 정의적 영역에 있어서의 변화를 야기하며, 이를 통해 실 제 임상상황을 분석하고 토론하며 자율적으로 학습함으로써 학습동기가 향상되는 것으로 보고하고 있다[3,6].

하지만 이와는 달리 PBL을 하는 동안 시간할당과 과제에 대한 부담감, 그룹 활동에 대한 어려움, 위축감 등을 경험하거 나[7,8], 학습의 자기주도성이 증진되지 않고[9], 비판적 사고 에 대한 긍정적인 효과가 나타나지 않는다는 보고도 있다 [1,10]. 이러한 상반된 결과들은 많은 부분 각 연구들이 수행 되는 상황, 즉 연구대상, 실시기간, 전공 등의 차이에 의한 것 으로 일부 해석될 수 있을 것이다.

한편, 연구방법 측면에서 PBL에 의한 학습자의 정의적 특 성의 변화는 양적인 결과만으로는 알기 힘들며, 질적인 분석 이 병행되어야 할 필요도 있다. 또한 $\mathrm{PBL}$ 의 효과를 극대화하 여 인지적인 지적 능력 외에도 정의적 영역에서의 학습 능력 이나 동기의 변화가 실제로 일어나기 위해서는 어느 정도의 기간으로 운영되는 것이 좋은지에 대한 조사도 필요하다. 따 라서 저자 등은 $\mathrm{PBL}$ 의 실시기간에 따라 $\mathrm{PBL}$ 이 의학전문대 학원생의 학습동기와 자기주도학습 준비도에 미치는 효과가 어떠한 차이가 있는지를 알아보고자 하였다. 또한 PBL의 효 과를 학습자가 직접 작성한 성찰일지의 내용분석을 통한 질 적 접근을 시도함으로써, $\mathrm{PBL}$ 의 효과를 극대화하기 위한 올 바른 PBL 시행 방안을 모색하고자 하였다.

\section{대상 및 방법}

\section{1. 연구대상 및 연구설계}

2007년 12월 2 5주 동안의 PBL에 참여하고 설문에 응답 한 의학전문대학원(의전원) 1 학년 100 명과 2학년 학생 90 명, 총 190 명을 대상으로 하였다. 학생들은 조당 11 명 이내로, 학 년당 각 12 조로 편성되었다. 1 학년은 2 개의 모듈로 2 주간, 2 학년은 5 개의 모듈로 5 주간 $\mathrm{PBL}$ 을 시행하였다. 각 모듈은 1 주일에 2번의 만남과 자율학습으로 진행되었다. 1학년은 $\mathrm{PBL}$ 시작 직전과 2주간의 $\mathrm{PBL}$ 종료 직후에, 2학년은 $\mathrm{PBL}$ 시작 직전과 5 주간의 $\mathrm{PBL}$ 종료 직후에 각 1 회씩 학년별 총 2 회의 학습동기검사와 자기주도적 학습준비도검사를 실시하 였다. 학년별 $\mathrm{PBL}$ 이 진행되는 전 과정에서 학생들이 느끼는 만족도와 $\mathrm{PBL}$ 의 효과에 대해 성찰일지에 자유롭게 서술하도 록 하였다.

\section{2. 연구도구}

학습동기는 $\operatorname{Kim}[11]$ 의 표준화된 학습동기검사를 사용하 여, 학습자가 자신의 수행능력에 대해 보이는 기대나 신념인 '학업적 자기효능감(academic self-efficacy)'과 자신의 실패 경험에 대해 건설적인 혹은 비건설적인 반응 여부를 알아보 는 '학업적 실패내성(academic failure tolerance)'을 평가하 였다. 신뢰도(Cronbach' $\alpha$ )는 0.94였다. 학업적 자기효능감 에는 자신감, 자기조절효능감, 과제수준 선호 등의 하위 변인 을, 학업적 실패내성에는 감정, 행동, 과제난이도 선호 등의 하위 변인을 포함하였다. 학업적 자기효능감은 자신의 수행 능력에 대해 보이는 기대나 신념을 의미하고, 학업적 실패 내 성은 자신의 실패 경험에 대하여 건설적으로 반응하는지 혹 은 비건설적으로 반응하는지를 나타낸다. 또한, 자신의 전반 적인 학문적 수행능력 및 학습능력에 대해 보이는 신념 정도 를 평가하는 자신감, 개인이 자기관찰, 자기판단, 자기반응과 같은 자기조절적 기제를 잘 수행할 수 있는지에 대한 효능기 대를 평가하는 자기조절효능감, 도전적이고 구체적인 목표를 선택하려 하는지에 대한 과제수준 선호의 하위 변인을 포함 한다. 반면, 학업적 실태 내성 척도는 실패 경험 후 보이는 감 
정반응이 긍정부정 정도를 평가하는 감정 요인과 실패 경험 후 이를 만회하기 위한 계획 수립, 방안 강구 정도 등의 구체 적이고 현실적 극복 행동 정도를 보는 행동 요인, 개인이 학습 과제난이도에 대해 보이는 인지 정서 행동 측면을 평가하는 과제난이도 선호 요인으로 구성된다.

자기주도학습 준비도 검사는 Guglielmino [12]의 자기주 도학습 준비도 검사(self-directed learning readiness scale, $\mathrm{SDLRS}$ )를 번안하여 만든 교사용 자기주도성 측정도구(selfdirected learning inventory for teacher) [13]를 사용하였 으며, 이는 학습에 대한 독창적 접근, 탐구적 접근, 자기 스스 로 계획을 세워 실천하는 자발적 계획, 학습 과정과 결과에 대 한 책임감, 지속적으로 학습하고자 하는 욕구와 학문 탐색을 즐기는 열정과 자신의 미래를 생각하며 어려운 상황을 문제 가 아닌 도전으로 대처하는 미래 지향성, 학습에 대한 자신의 통제 능력에 대한 확신 등 총 58문항 7 개의 하위 변인으로 구 성된다. 전체 신뢰도는 0.90 이었다.

\section{3. 통계분석}

$\mathrm{PBL}$ 사전과 사후의 학습동기와 자기주도학습 준비도 차이 는 paired t-test를 사용하여 검증하였다. PBL 기간에 따른 차이, 즉 2 개의 모듈(2주간)을 실시한 1학년과 5 개의 모듈(5 주간)을 실시한 2학년의 학습동기 및 자기주도학습 준비도 차 이는 비교하고자 하는 연구대상의 학년이 다르기 때문에 사
전검사 점수가 미치는 영향을 최대한 배제하기 위하여 사전 검사 점수를 공변인으로 하는 반복측정 MANCOVA로 검증 하였다. 성찰일지는 1학년 24편, 2학년 120편으로 전체 144 편을 읽고 분류작업을 거쳐 의미단위를 추출한 후, 의미단위 의 타당성을 전문가 2 인에게 검증을 받은 후, $\mathrm{PBL}$ 의 효과, $\mathrm{PBL}$ 의 진행에 있어서의 어려움, $\mathrm{PBL}$ 만족도의 영역으로 나 누어 재분석하였다. 통계분석은 SPSS 12.0 for Windows (SPSS Inc., Chicago, USA) 프로그램을 사용하였으며, 통계 적 유의성은 유의수준 $5 \%$ 로 정하였다.

\section{결과}

\section{PBL이 학습동기와 자기주도학습 준비도에 미치 는 영향}

\section{1) 학습동기}

1 학년 학생의 경우 학업적 자기효능감의 하위 변인으로 자 기조절효능감, 과제수준선호에서 유의한 증진 효과가 나타났 으며 자신감은 줄었다. 학업적 실패내성 영역의 하위 변인으 로 감정에서 감소효과를, 과제난이도선호에서 유의한 증진효 과가 나타났다. 학습동기 전체 점수에 있어서 차이는 없었다. 반면, 2 학년 학생의 경우 학업적 자기효능감 전체점수와 그

Table 1. Effects of Problem-Based Learning on Learning Motivation

\begin{tabular}{|c|c|c|c|c|c|c|c|c|}
\hline \multirow[b]{2}{*}{ Variables } & \multicolumn{3}{|c|}{ Year $1(n=100)$} & \multicolumn{3}{|c|}{ Year $2(n=90)$} & \multirow[b]{2}{*}{$p$-value ${ }^{a l}$} & \multirow[b]{2}{*}{ p-value ${ }^{b l}$} \\
\hline & Pre-PBL & Post-PBL & $\begin{array}{c}\triangle \% \\
\text { change }\end{array}$ & Pre-PBL & Post-PBL & $\begin{array}{c}\triangle \% \\
\text { change }\end{array}$ & & \\
\hline Academic self-efficacy & $90.9 \pm 10.3$ & $93.1 \pm 10.1$ & $3.5 \pm 14.6$ & $90.7 \pm 15.0$ & $95.0 \pm 11.0$ & $10.0 \pm 36.8$ & $0.056, \quad 0.018^{c /}$ & $0.013^{\mathrm{cl}}$ \\
\hline Confidence & $26.1 \pm 5.7$ & $24.3 \pm 5.8$ & $-5.5 \pm 19.0$ & $24.2 \pm 6.9$ & $23.9 \pm 6.6$ & $3.6 \pm 37.1$ & $<0.001^{c l}, \quad 0.587$ & $0.018^{\mathrm{cl}}$ \\
\hline Self-control efficacy & $37.3 \pm 6.0$ & $40.1 \pm 6.0$ & $9.7 \pm 22.8$ & $38.4 \pm 8.6$ & $41.6 \pm 6.4$ & $15.8 \pm 45.5$ & $<0.001^{c \mid},<0.001^{c l}$ & $0.017^{c l}$ \\
\hline Task-level preference & $27.5 \pm 3.8$ & $28.7 \pm 3.5$ & $5.7 \pm 15.5$ & $28.0 \pm 5.2$ & $29.5 \pm 3.4$ & $11.9 \pm 46.0$ & $<0.001^{c \mid}, \quad 0.007^{c l}$ & $0.017^{\mathrm{cl}}$ \\
\hline Academic failure-resistance & $64.9 \pm 8.6$ & $66.0 \pm 8.8$ & $2.8 \pm 15.5$ & $65.6 \pm 10.4$ & $67.9 \pm 8.0$ & $6.7 \pm 25.8$ & $0.229, \quad 0.043^{c 1}$ & $0.041^{c l}$ \\
\hline Feeling & $26.1 \pm 5.7$ & $24.3 \pm 5.8$ & $-0.9 \pm 27.0$ & $22.8 \pm 5.7$ & $22.1 \pm 5.5$ & $-0.3 \pm 22.8$ & $<0.001^{c l}, \quad 0.139$ & 0.613 \\
\hline Behavior & $20.5 \pm 5.4$ & $21.8 \pm 6.5$ & $7.3 \pm 17.3$ & $20.4 \pm 3.8$ & $21.9 \pm 3.2$ & $10.0 \pm 20.8$ & $0.237,<0.001^{c)}$ & 0.188 \\
\hline Task-difficulty preference & $20.5 \pm 3.4$ & $21.7 \pm 3.3$ & $6.1 \pm 27.0$ & $22.4 \pm 5.3$ & $23.9 \pm 3.6$ & $21.1 \pm 80.0$ & $<0.001^{c \mid}, \quad 0.012^{c l}$ & $0.001^{c l}$ \\
\hline Total & $155.8 \pm 17.1$ & $159.1 \pm 16.7$ & $3.1 \pm 13.8$ & $156.3 \pm 24.3$ & $162.9 \pm 16.8$ & $8.3 \pm 30.9$ & $0.085, \quad 0.020^{c 1}$ & $0.016^{c l}$ \\
\hline
\end{tabular}

Data are means $\pm S D . \triangle \%$ change, the change divided by the baseline value, multiplied by 100 .

$\mathrm{PBL}$ : Problem-based learning.

${ }^{a)}$ Paired t-test for within group (p-value in Year 1, p-value in Year 2), ${ }^{b)}$ MANCOVA of differences between groups, ${ }^{c /} p$-value $<0.05$. 
So Jung Yune, et al : Effects of Differences in Problem-Based Learning Course Length on Academic Motivation and Self-Directed Learning Readiness in Medical School Students

Table 2. Effects of Problem-Based Learning on Self-Directed Learning Readiness

\begin{tabular}{|c|c|c|c|c|c|c|c|c|}
\hline \multirow[b]{2}{*}{ Variables } & \multicolumn{3}{|c|}{ Year $1(n=100)$} & \multicolumn{3}{|c|}{ Year $2(n=90)$} & \multirow[b]{2}{*}{$p$-value ${ }^{a l}$} & \multirow[b]{2}{*}{$p$-value ${ }^{b /}$} \\
\hline & Pre-PBL & Post-PBL & $\begin{array}{c}\triangle \% \\
\text { change }\end{array}$ & Pre-PBL & Post-PBL & $\begin{array}{l}\triangle \% \\
\text { change }\end{array}$ & & \\
\hline Originative characteristics & $32.5 \pm 3.9$ & $33.0 \pm 3.6$ & $2.5 \pm 12.8$ & $32.9 \pm 4.0$ & $33.1 \pm 5.1$ & $1.1 \pm 15.6$ & $0.242, \quad 0.781$ & 0.735 \\
\hline Investigative characteristics & $10.5 \pm 1.8$ & $10.6 \pm 1.9$ & $3.5 \pm 21.6$ & $10.4 \pm 2.2$ & $10.4 \pm 2.2$ & $2.5 \pm 21.8$ & $0.557, \quad 0.959$ & 0.995 \\
\hline Voluntary planning & $15.2 \pm 1.9$ & $15.0 \pm 2.5$ & $-0.6 \pm 15.1$ & $15.1 \pm 2.4$ & $15.1 \pm 2.5$ & $1.2 \pm 17.7$ & $0.402, \quad 0.964$ & 0.611 \\
\hline Responsibility to learning & $14.3 \pm 2.0$ & $14.9 \pm 1.7$ & $5.5 \pm 15.7$ & $15.0 \pm 1.9$ & $15.3 \pm 1.9$ & $3.1 \pm 12.3$ & $0.009^{c 1}, 0.066$ & 0.474 \\
\hline Eager to learning & $26.8 \pm 2.7$ & $26.7 \pm 2.8$ & $0.1 \pm 10.7$ & $26.8 \pm 3.4$ & $26.9 \pm 3.2$ & $1.0 \pm 11.0$ & $0.684,0.776$ & 0.649 \\
\hline Future orientation & $15.2 \pm 1.8$ & $15.6 \pm 1.8$ & $2.7 \pm 10.1$ & $15.3 \pm 1.8$ & $15.9 \pm 2.1$ & $4.2 \pm 14.3$ & $0.039^{\mathrm{cl}}, 0.017^{\mathrm{cl}}$ & 0.257 \\
\hline Self-confidence in learning & $22.7 \pm 2.6$ & $23.1 \pm 2.7$ & $2.7 \pm 12.6$ & $23.0 \pm 2.6$ & $23.0 \pm 3.5$ & $0.7 \pm 16.3$ & $0.134,0.953$ & 0.825 \\
\hline Total & $137.4 \pm 9.0$ & $138.8 \pm 9.3$ & $1.2 \pm 6.6$ & $138.6 \pm 9.7$ & $139.7 \pm 11.9$ & $1.0 \pm 8.3$ & $0.131,0.378$ & 0.841 \\
\hline
\end{tabular}

Data are means $\pm S D . \triangle \%$ change, the change divided by the baseline value, multiplied by 100 .

PBL: Problem-based learning.

${ }^{\text {al }}$ Paired t-test for within group (p-value in Year 1, p-value in Year 2), ${ }^{\text {b) }}$ MANCOVA of differences between groups, ${ }^{c)} \mathrm{p}$-value $<0.05$.

하위 변인으로 자기조절효능감, 과제수준선호에서 유의한 증 진 효과가 나타났다. 학업적 실패내성 영역에서도 전체점수 와 그 하위 변인으로 행동과 과제난이도선호에서 유의한 증 진효과가 나타났다. 그리고 학습동기 전체 점수에 있어서도 유의한 증진 효과를 보였다(Table 1).

\section{2) 자기주도적 학습 준비도}

1 2학년 모두에서, PBL은 자기주도 학습준비도에 전체적 으로는 유의미한 영향을 미치지 않았다. 하지만, 1 학년에서 그 하위 변인 중에 학업에 대한 책임감과 미래지향성에서, 2 학년 에서는 미래지향성에서 유의한 증진 효과를 보였다(Table 2).

\section{PBL 기간에 따른 학습동기와 자기주도학습 준비 도의 변화 차이}

5 주간의 PBL을 시행한 2학년이 2주간의 PBL을 시행한 1 학년보다 학습동기 전체와 그 하위 변인으로 자신감, 자기조 절효능감, 과제수준선호 모두에서 보다 유의한 증대효과를 나타내었다. 학업적 실패내성 영역에서는 전체점수와 그 하 위 변인 중에 과제난이도선호에서만 2학년이 1학년보다 더욱 유의한 증진효과를 보였다(Table 1). 반면, PBL 기간에 따른 자기주도적 학습 준비도에 있어서는 전체적으로나 각각의 하 위 변인 모두 유의한 차이가 나타나지 않았다(Table 2).

\section{3. 성찰일지 분석결과}

\section{1) $\mathrm{PBL}$ 효과}

PBL을 통해 학습을 통한 내용의 이해뿐 아니라, 이론적 내 용의 실제적 적용에 대한 이해 역시 높아졌다. 학생들은 토론 또는 협동학습의 장점을 체험적으로 알 수 있었다. 혼자 공부 할 때보다 더 많은 양의 학습이 가능했으며, 상대방의 관점을 이해하여 사고의 폭이 넓어졌다. 스스로의 학습을 되돌아보 고, 자신의 학습에서 알고 있는 것과 잘 알지 못하는 것을 구 분할 수 있었으며, 이러한 과정을 통해 학습동기가 증진되고 있었다.

\section{2) PBL 진행에 있어서의 어려움}

학생들이 처음에는 토론 상황에 익숙지 못한 경우가 많았지 만, $\mathrm{PBL}$ 을 통해 점차적으로 토론과 발표에 익숙해져 가는 양 상을 보였다. $\mathrm{PBL}$ 은 토론과 발표에 있어서의 학습자 태도를 긍정적으로 변화시키고 있었다. 그리고, $\mathrm{PBL}$ 을 진행하는 사 회자나 튜터의 역할에 대해 학생들은 다소 불만을 표시하였는 데, 토론이 침체되었을 때 혹은 지나친 논쟁이 일어날 때 사회 자의 적절한 개입은 요구되나 미흡한 경우도 있었고, 너무 지 나친 개입이 있을 때는 자발적인 토론과 발표가 위축되는 경 향을 보인 경우에 해당된다. 가설과 학습문제 도출에 있어 기 본적인 지식의 부족이 가장 문제가 되는 것으로 나타났다. 


\section{3) $\mathrm{PBL}$ 만족도}

$\mathrm{PBL}$ 방식이 힘든 면은 있었으나, 도움이 된다는 반응들이 대부분이었다.

\section{고찰}

본 연구는 PBL이 의학전문대학원생의 학습동기와 자기주 도학습 준비도에 어떠한 영향을 미치는지, 그리고 PBL의 시 행 기간에 따른 효과의 차이가 있는지를 알아보기 위한 목적 으로 진행되었다. 그 결과, $\mathrm{PBL}$ 은 1 2학년 모두 의학전문대 학원생의 학습동기의 학업적 자기 효능감 영역 중 자기조절 효능감과 과제수준 선호에서 유의한 증진효과를 보였으며, 학업적 실패내성 영역에서는 과제난이도 선호의 하위 변인에 서 증진 효과를 나타내었다. 2학년의 경우에는 학업적 실패내 성 영역 중 행동에서 유의한 증진이 나타났다(Table 1). 즉, 개인이 스스로 자기조절적 기제를 잘 수행할 수 있는지에 대 한 자기 효능에 대한 기대가 높아졌으며, 도전적이고 구체적 인 과제를 스스로 선택하고자 하는 선호도 역시 높아졌음을 알 수 있다. 그리고 실패 경험 후에 이를 구체적이고 현실적인 방법으로 극복하고자 하는 행동에 대한 선호도가 높아졌으며, 특히, 과제난이도에 대해 보이는 인지 정서 행동적 측면이 긍 정적으로 변화되었음을 알 수 있다. 또한 자기주도학습 준비 도에 있어서는 학업에 대한 책임감과 미래지향성 변인에서의 유의미한 증진 효과를 보였다. 그러나 PBL을 통해 자기주도 학습 준비도에 있어서의 전체적인 증진 효과는 나타나지 않 았다. 반면 1, 2학년 모두 자기주도학습 준비도의 하위 변인 중 미래지향성에 있어서는 유의한 증진 효과를 보였다. 이러 한 점에서 볼 때, 학생들은 PBL 상황에서 겪게 되는 다양한 정서적 인지적 경험들로 인해 최소한 어려운 상황을 문제가 아닌 도전으로 간주하고 이를 대처하고자 하는 평생 학습자 로서의 태도는 기를 수 있음을 알 수 있다(Table 2).

$\mathrm{PBL}$ 을 통해 학생들에게 나타난 변화는 PBL을 통해서 학 습자의 자기주도성과 학습동기 등의 긍정적인 변화가 나타났 다고 밝힌 선행연구 $[1,2,4,5,14]$ 들의 결과와 유사하다고 할 수 있다. 또한 PBL이 전통적인 수업방식에 비해 효과적인 방법 임을 밝힌 여러 연구[15,16,17]와도 같은 맥락에서 생각해 볼
수 있는 결과이다.

그리고 이러한 긍정적인 변화는 본 연구에서 분석한 성찰 일지의 내용 분석에서도 마찬가지의 결과로 나타났다. 학생 들은 PBL을 통해 학습 내용의 이해도가 증진되었고, 토론이 나 협동학습 등의 장점을 인식할 수 있게 되었으며, 자신을 성 찰하고 학습동기를 증진하게 되었다고 보고하였다. 특히, 수 동적인 강의식 수업이 아니라 능동적인 학습자 중심의 수업 과 실제적인 문제를 통해 종합적이고 통합적인 학습이 가능 하게 되었으며, 사고의 폭이 넓어졌으며, 자신이 알고 있는 것 과 모르는 것에 대한 정확한 이해가 이루어질 수 있었다는 면 에서 $\mathrm{PBL}$ 이 효과적이었다는 반응을 보였다. 하지만, $\mathrm{PBL}$ 진 행에 있어서의 어려움으로 PBL을 진행하는 사회자나 튜터의 역할에 대한 불만을 제기한 경우도 있었다. 이는 집단의 역동 을 원활하게 유지하고, 활발한 토론을 이끌어가는 사회자의 역할과 모듈에서 제시하는 문제 해결의 방향이 자칫 엉뚱하 게 흘러가지 않도록 하는 튜터의 역할을 기대하고 있었기 때 문으로 판단된다. 또한, 기본적인 지식이 부족하다고 판단하 여 가설과 학습과제도출이 가장 어려웠다고 하였지만, 이러 한 과정을 통해서 스스로 자신이 알고 있는 것과 부족한 부분 에 대해 인식하고, 학습에 대한 동기가 높아진 것 같다고 학생 들은 기술하였다.

따라서 PBL은 학생들의 인지적 능력의 향상뿐만 아니라, 정의적인 학습에 대한 효능감과 실패에 대한 내성을 기르는 데에도 효과적이며, 스스로 자신의 학습을 주도해 나갈 수 있 는 자기주도학습 준비도를 기르는 데에도 일부 도움이 된다 는 것을 알 수 있다.

그러나 학습동기의 여러 변인 중 자신감과 실패에 대한 감 정 요인에서 효과가 나타나지 않은 것은 선행연구[8]에서도 보고된 바 있는 결과로, $\mathrm{PBL}$ 이 운영되는 기본 절차인 토론과 발표과정 등에 대한 두려움이나 PBL을 통해 학습문제를 도 출하고 문제해결을 위해 추론해 나가는 상황에서 스스로의 지식이 부족함을 확인하게 되는 데에서 나타난 결과로 해석 될 수 있다. 따라서 학생들이 PBL을 보다 부담 없이 받아들 이고, 이를 통해 학습의 긍정적인 면들을 더 잘 발전시키기 위 해서는 지식의 습득이나 PBL 훈련뿐만 아니라, 토론법, 발표 법, 협동학습의 기본적 기술을 익히도록 하는 것이 도움이 될 수 있으며, 문제해결방법이나 문제발견방법에 대한 훈련 또 
So Jung Yune, et al : Effects of Differences in Problem-Based Learning Course Length on Academic Motivation and Self-Directed Learning Readiness in Medical School Students

한 이루어져야 할 필요가 있을 것이다. 이를 통해, $\mathrm{PBL}$ 상황 에서 겪을 수 있는 실패 경험을 정서적으로 잘 극복할 수 있도 록 도와줄 수 있을 것으로 기대된다.

$\mathrm{PBL}$ 의 시행에 있어 어느 정도의 기간 동안 운영해야 할 것 인가에 대해서는, 적어도 6주 이상은 시행해 보아야 제대로 경험할 수 있다고 Barrows와 Tamblyn 교수가 보고[18]한 바 있으나, 이에 대한 국내 연구는 그리 많지 않다. 단지, PBL이 한 과목만으로 적용되거나 적용기간의 부족으로 인해 $\mathrm{PBL}$ 의 효과가 나타나지 않았다는 선행연구 $[1,10]$ 와 통합교육과정 내에서 7주간 간호학생을 대상으로 PBL을 진행한 결과 메타 인지 및 자기효능감, 학습동기에 있어서는 효과가 나타났다 는 연구[14] 등에 근거할 때, 보다 장기간의 운영이 PBL의 교 육목적 달성에 결정적인 한 요소가 될 것으로 추정된다. 본 연 구에서도 5 주간의 $\mathrm{PBL}$ 의 운영이 2주간에 비해 학습동기에 있어서 유의한 효과의 증대를 보였다. 즉, 학습동기 전체와 그 하위 변인으로 자신감, 자기조절효능감, 과제수준선호 모두에 서 더욱 유의한 증대효과를 나타내었고, 학업적 실패내성 영 역에서도 전체점수와 그 하위 변인 중에 과제난이도선호에서 더욱 유의한 증진효과를 보였다. 하지만, 자기주도학습 준비 도에는 전체적으로 영향을 미치지는 않았다. 물론, 시행 기간 이외에도 $\mathrm{PBL}$ 모듈의 구성과 진행 방법, $\mathrm{PBL}$ 사례의 적절성, 튜터 역할 교육, 학생의 토론 참여도, $\mathrm{PBL}$ 집단 내에서의 역 동 등의 다른 요인들도 많은 영향을 미칠 것이기 때문에, 본 연구 결과를 토대로 최소한 5주 이상 PBL을 운영해야 한다고 단정 지을 수는 없다. 결론적으로, $\mathrm{PBL}$ 은 학생들의 학습동기 와 자기주도학습 준비도를 증진시키는 데 효과적인 교수-학 습 방법인 것으로 나타났다. 게다가 본 연구 결과에 의하면 $\mathrm{PBL}$ 의 효과적인 시행을 위해서는 5 주 이상의 보다 장기간 시 행이 중요한 역할을 할 수 있을 것으로 추정된다.

그러나 본 연구는 2 주와 5 주 동안의 PBL 경험만을 비교하 였으며, 동일한 학년을 대상으로 연구하지 못했다는 점에서 본 연구 결과를 일반화시키기에는 제한점이 있다. 또한, 문제 바탕학습의 학습효과증진을 위한 튜터의 역할이 상당히 중요 하므로, 정기적인 튜터교육을 시행하여 튜터의 표준화를 의 도하였다. 하지만, 학생들의 반응처럼 튜터 역할에 대한 불만 족이 있는 경우가 있었다. 이 점 또한 연구의 제한점으로 작용 할 수 있으나, 학년별 만족스럽지 못한 튜터는 1 2명에 불과
하여 학년 간의 비교에 미치는 영향은 미흡하였을 것으로 판 단된다. PBL의 조별 인원을 편성할 때 조당 5 7명의 학생이 적절하다고 알려져 있으나, 본 연구에서는 튜터의 부족으로 부득이 11 명 이내의 학생으로 조를 구성하였다. 하지만, 상대 적으로 많았던 조원이었음에도 불구하고 PBL의 긍정적 기대 효과는 관찰할 수 있었다는 점에서 의미가 있을 수 있겠다. 추 후 동일한 학년을 대상으로 PBL의 시행 방법에 따른 효과를 알아보는 연구가 계속적으로 진행되어야 할 필요가 있으며, PBL의 효과를 극대화하기 위해 PBL 진행에 영향을 미치는 제반 요인들이 PBL에 어떠한 영향을 미치는지에 대해서는 보다 구체적이고 심층적인 연구가 추후에 이루어져야 할 것 으로 생각된다.

\section{REFERENCES}

1. Bae YS, Lee SH, Kim MH, Sun KS. Effects of PBL (problem based learning) on self-directed learning and critical thinking disposition of nursing students. J Korean Acad Soc Nurs Educ 2005; 11: 184-190.

2. Lee SH, Kim MH, Sun KS. The clinical competence and related factors of nursing students: focused on the subjects who studies problem-based learning. J Korean Acad Adult Nurs 2007; 19: 753-762.

3. Hwang YY, Chu MS, Park CS. The effects of problem based leaning (PBL) in nursing students studying through PBL curriculum. J Korean Acad Soc Nurs Educ 2007; 13: 155-161.

4. Hwang SY, Kim MJ. A comparison of problem-based learning and lecture-based learning in an adult health nursing course. Nurse Educ Today 2006; 26: 315-321.

5. Tiwari A, Chan S, Wong E, Wong D, Chui C, Wong A, Patil N. The effect of problem-based learning on students' approaches to learning in the context of clinical nursing education. Nurse Educ Today 2006; 26: 430-438.

6. Cooke M, Moyle K. Students' evaluation of problem- 
based learning. Nurse Educ Today 2002; 22: 330-339.

7. Barrow EJ, Lyte G, Butterworth T. An evaluation of problem-based learning in a nursing theory and practice module. Nurse Educ Pract 2002; 2: 55-62.

8. Lee CY, Cho WJ, Storey MJ, Kim E, Lee KH, Bae SH, Kim GS, Seo KM, Ham OK. Evaluation research on the application of problem-based learning program in community health nursing. J Korean Community Nurs 2003; 14: $1-8$.

9. Williams B. Self direction in a problem based learning program. Nurse Educ Today 2004; 24: 277-285.

10. Choi H. The effects of PBL (problem based learning) on the metacognition, critical thinking, and problem solving process of nursing students. J Korean Acad Nurs 2004; 34: $712-721$.

11. Kim AY. Learning self efficacy: theory and field research. Seoul, Korea: Hakjisa; 2007.

12. Guglielmino LM. Development of the self-directed learning readiness scale [dissertation]. Athens, USA: University of Georgia; 1977.

13. Kim GZ, Kim KS, Yoo KO, Yoo GH. Development and utilization plan of self-directed learning inventory for teacher. Sahoegyoyukhagyeongu 1996; 2: 1-25.

14. Hwang SY. The effects of PBL (problem based learning) on academic achievement, critical thinking ability, learning attitude, and motivation [dissertation]. Gwangju, Korea: Chunnam University; 2003.

15. Gallagher S, Stepien WJ. Implementing problem-based learning in science classrooms. Sch Sci Math 1995; 95: 136-146.

16. Sage SM. A qualitative examination of problem-based learning at the K-8 level: preliminary finding. Paper presented at: The Annual Meeting the American Educational Research Association; 1996 April 8-12; New York, USA.

17. Dochy F, Segers M, Van den Bossche P, Gijbels D. Effects of problem-based learning: a meta-analysis. Learn Instr 2003; 13: 533-568.

18. Barrows HS, Tamblyn RM. Problem-based learning: an approach to medical education. New York, USA: Springer Publishing Company; 1980. 
Appendix. 성찰일지에서 나타난 PBL 효과

1. $\mathrm{PBL}$ 효과

1) 학습내용에 대한 이해

- 평소에 모르고 넘어간 기초적인 부분에 대해 다시 한 번 자세하게 알 수 있었으며, 앞으로 어떤 식으로 공부를 해나가야 할지에 대해서도 알 수 있었다. (2학년)

- 호흡기내과 수업을 2학년 1학기 때 배워서, 그 내용이 잘 생각나지 않았다. 그런데 오늘 토론을 하고 다시 한 번 자료를 보게 되면서 복습할 수 있게 되어 많은 도움이 되었다. (2학년)

- 지금까지 배워온 단편적인 지식들을 통합하여 접근하는 방법을 배우게 된 것 같다. (1학년)

- 단지 병을 추정하는 것이 아니라, 근거에 기초하여 추론하는 과학적 사고가 필요함을 깨달았다. (2학년)

- 처음으로 환자의 병을 추측해 보면서, 지금까지 배운 기초와 임상과목들을 되짚을 수 있었다. 그리고 이러한 이론들이 어떤 영향을 미치는지, 임상에서 어떻게 적용될 수 있는지를 조금이나마 알 수 있었다. (1학년)

2) 토론/협동학습에 대한 이해

- 계속되는 토론 수업을 하면서 토론에 임하는 자세나 방식들이 얼마나 중요한지 다시 한 번 깨닫게 되었다. 예전에는 이런 방식에 익숙하지 않았던 것에 비해 놀라운 변화가 일어났다. (2학년)

- 서로 잘 모르는 시술이나 검사에 대해 아는 사람이 설명해주면서 토론한 것이 매우 좋았다. 잘 모르는 부분은 서로 가르쳐 주며 공부하는 것이 정말로 큰 도움이 되었다. (2학년)

- 내가 준비한 학습과제 외에 다른 학우가 준비한 학습과제까지 공부할 수 있어 이해가 더 빠르고 쉽게 결과를 도출해 낼 수 있어 좋았다. (2학년)

3) 자아성찰 및 학습동기 증진

- 환자의 치료 및 처치 후 양상을 보면서 빠른 진단 및 처치의 중요성을 깨닫게 되었고, 더 열심히 공부해야겠다는 생각을 하게 되었다. (2학년)

- 솔직히 임상 수업을 들은 후라 나의 실력이 많이 향상되었을 거라는 기대를 가지고 있었다. 그러나 오늘 PBL 수업을 하면서, 아직 많이 부족함을 느겼다. 내가 가지고 있는 의학적 지식수준과 정도를 아는 데 도움이 되었으며, 앞으로 더 열심히 공부해야겠다 는 것을 느겼다. (2학년)

- 확실히 알지 못하는 것이 아예 모르는 것보다 더 위험할 수 있다는 생각이 들었다. 배웠으나 정확히 모르고 있는 부분이 무엇인지 알 수 있어 도움이 되었다. (1학년)

- 많은 정보들 가운데에서 더욱 중요하고 덜 중요한 것들을 구분할 수 있는 능력이 의사에게는 반드시 필요하다는 것을 느겼다. (1학년)

2. $\mathrm{PBL}$ 진행에 있어서의 어려움

1) 토론 상황에서의 학습자의 태도

- 토론의 방향이 너무 한 방향으로 치우쳐서 진행되었다. 다들 정신과 질환에 대한 지식이 너무 부족해서 그런지, 모두들 기질적 문제 또는 신체 이상에만 초점을 맞추는 경향이 있었다. (2학년)

- 자연스러운 진행을 위해 자유롭게 이야기하다 보니, 같은 생각을 갖고 있어도 발표하지 못하는 경우가 종종 생겼다. 계속 한 사람이 이야기하는 것에 대한 규제가 필요할 것 같다. (2학년)

- 토론을 하는 중에 공격적인 태도를 보이거나 혹은 상대방이 말한 것을 반복해서 말하는 경우가 있었다. 자기 의견을 내는 것도 중요하지만 경청하는 자세도 필요한 것 같다. (2학년)

2) 사회자 또는 튜터의 역할

- 토론이 침체되었을 때 적절하게 토론을 이끌어 갈 수 있는 사회자의 역할이 필요한 것 같다. 무슨 애기를 언제 해야 하는지 알지 못해서 힘들었다. (2학년)

- 비슷한 수준의 지식을 가진 1 학년들이 의견을 나누다 보니 종종 한계에 부딪히게 되었는데, 이때 튜터의 적절한 방향제시가 있다면 보다 풍부한 PBL 시간을 가질 수 있었을 것이다. (1학년)

- 튜터 교수님이 토론 중 나왔던 과제와는 다른 새로운 학습 과제를 설정해 주셨다. 나중에 퀴즈에는 도움이 될지 모르겠지만 허탈한 느낌을 받았다. (2학년)

- 너무나 다양한 의견들이 나온 가운데 합의가 쉽지 않은 부분이 있었다. 사회자나 튜터가 적극적으로 중재하거나 혹은 학생들 자신의 생각이 틀릴 수 있다는 것을 인지하고 유연하게 토론을 이끌어가야 할 것이다. (1학년)

3) 가설과 학습문제 도출과 추론

- 가장 가능한 가설을 세우기가 힘들었다. 임상에 대한 지식이 부족해서일지도 모르지만, 너무나 다양한 병들이 해당되는 것 같아서 자료를 받고서도 여러 가설들이 떠올라 혼란스러웠다. (1학년)

- 핵심과 학습목표에 맞는 가설 설정, 그리고 학습과제 도출이 아직도 미흡한 것 같다. (2학년)

- 기본적으로 너무 임상적 질환에 대해서 질환명을 알아내는 데 치중하다 보니, 정작 중요하게 짚고 넘어가야 할 문제들을 간과하게 
된 것 같다. (2학년)

- 학습과제에만 충실하다 보니, 퀴즈의 내용을 풀어가기가 어려웠다. 퀴즈는 기본적인 문제에 중점을 둔 문제가 나왔고, 우리가 공부한 학습과제와는 거리가 있었다. (2학년)

- 질병의 진단이 너무 일찍 이루어지면서, 그 질병에만 초점이 맞춰졌다. 다양한 학습과제를 도출하고 추론하는 데 어려움이 있었다. (2학년)

3. PBL 만족도

- 매우 만족한다. 일방적인 정보전달에 그친 교실 수업과는 다르게 구성원 스스로가 해답을 찾아가는 점이 새롭고 도움이 되었다. (1학년)

- 매우 만족한다. 여러 사람들이 각자 공부한 내용을 토대로 하나의 결론을 이끌어 낸다는 점이 너무 뿌듯하고 즐거웠다. 또한 자료를 모으고 공부하는 데 많은 시간이 걸렸지만, 이를 통해 많은 부분에 대해 보다 더 확실히 알 수 있었다. (1학년)

- 매우 만족한다. 사례에서 나온 임상증례를 추론하여 나름대로 병명을 생각해보고 가설을 설정하는 과정이 재미있고 도움이 되었다. (2학년)

- 매우 만족한다. PBL을 통해서 발표력이 향상되었고, 공부해야겠다는 의욕이 생겼다. (1학년)

- 매우 만족한다. 한편의 영화와도 같았다. (1학년) 